The aim of this study was to elaborate a new algorithm, based on serum CA125, HE4 and age, to assess the risk of malignancy in premenopausal patients with pelvic mass

Methodology The training dataset included 284 premenopausal patients operated because of presence of pelvic mass, out of which 35 and 249 had malignant and benign disease respectively. A novel algorithm, based on serum HE4, CA125 and patient's age as variables, has been developed by using the scenario of discrimination "benign diseases versus all stages of epithelial ovarian cancer (EOC) together with borderline ovarian tumors (BOT) FIGO stage 1c2-3c". This algorithm was named Risk of Ovarian Cancer Kazan Index (ROCK-I). The validating dataset consisted of consecutively operated premenopausal patients with pelvic mass out of which there were 187 cases of benign diseases, 20 EOC and 4 BOT FIGO stage $1 \mathrm{c} 2-3 \mathrm{c}$. An analysis with inclusion of BOT stage $1 \mathrm{a}-1 \mathrm{c} 1$ and non-EOC will be reported separately.

Result(s)* In the validating dataset the specificities of ROCK-I and ROMA were $92 \%$ and $85 \%$ respectively $(p<0.05)$. When the above-mentioned scenario of discrimination was used the sensitivities of ROCK-I and ROMA were 95.8\% and 91.7\% respectively, accuracies 92.4 and $85.8 \%$, positive predictive values $60.5 \%$ and $44 \%$ respectively. Areas under receiveroperating-characteristic curves (ROC-AUC) of ROCK-I, ROMA and CA125 were $0.984,0.94$ and 0.901 respectively. The difference in ROC-AUC between ROCK-I and CA125 was statistically significant $(p=0.03)$. A more detailed comparison of the performance of algorithms is shown in table 1 and figure 1 .

Abstract 1128 Table 1 Comparison of the performance of ROCKI and ROMA

\begin{tabular}{|c|c|c|c|c|c|c|c|c|}
\hline & \begin{tabular}{|l|} 
Sensitivity \\
(95\%) CDD.\%
\end{tabular} & $\begin{array}{l}\begin{array}{l}\text { Specificity } \\
(95 \% \% \text { CD. \% }\end{array} \\
\end{array}$ & \begin{tabular}{|l|} 
PPV \\
$(99 \%$ CD) $\%$ \\
\end{tabular} & $\begin{array}{l}\mathrm{NPV} \\
(95 \% \mathrm{CD}) \%\end{array}$ & $\begin{array}{l}\text { Accuracy } \\
(95 \% \text { CD) \% }\end{array}$ & $\begin{array}{l}\mathrm{LR+} \\
(95 \% \mathrm{CD})\end{array}$ & $\begin{array}{c}\text { DOR } \\
(95 \% \mathrm{CD})\end{array}$ & $\begin{array}{c}\text { ROC-AUC } \\
(99 \% \mathrm{CI})\end{array}$ \\
\hline & \multicolumn{8}{|c|}{$\begin{array}{l}\text { Training dataset } \\
\end{array}$} \\
\hline ROCK-I & $\begin{array}{c}99.6 \\
(75.3-96.0) \\
\end{array}$ & $\begin{array}{l}93.7^{*} \\
(90-96.1)\end{array}$ & $\begin{array}{l}66.7^{*} \\
(52.485)\end{array}$ & \begin{tabular}{|l|}
99.5 \\
$(96.0-99.4)$ \\
\end{tabular} & $\begin{array}{l}99.22^{*} \\
(89.795 .6)\end{array}$ & $\begin{array}{l}14.3^{*} \\
(8.8-23.2)\end{array}$ & $\begin{array}{l}11.28 .1 \\
(38.6424 .7) \\
\end{array}$ & $\begin{array}{l}0.997 \\
(0.94-0.992)\end{array}$ \\
\hline \multirow[t]{2}{*}{ ROMA } & $\begin{array}{l}83.9 \\
(68.92 .9\end{array}$ & $85.7^{*}$ & $45.2^{*}$ & 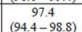 & $\begin{array}{c}85.5 * \\
(809-891)\end{array}$ & $\begin{array}{c}5.9^{*} \\
(42-83)\end{array}$ & $\begin{array}{l}311 \\
(118-820)\end{array}$ & 0.941 \\
\hline & \multicolumn{8}{|c|}{$\begin{array}{c}(35.7-5.2) \\
\text { Validating dataset }\end{array}$} \\
\hline ROCK-I & $\begin{array}{l}95.8 \\
(78.9-99.9)\end{array}$ & $\begin{array}{l}92.0^{*} \\
(88.1-95.4)\end{array}$ & $\begin{array}{c}60.5 \\
(48.4-71.5)\end{array}$ & $(96.2-99.9)$ & $\begin{array}{l}9.42 .4 \\
(88.0-95.0)\end{array}$ & $\begin{array}{c}12.0 \\
(7.3-19.0)\end{array}$ & $\begin{array}{l}203.7 \\
(33.3-2091.1)\end{array}$ & $\begin{array}{l}0.0984 \\
(0.969-0.998) \\
\end{array}$ \\
\hline ROMA & $\begin{array}{c}91.7 \\
(73.0-99.0)\end{array}$ & $\begin{array}{l}85.0^{*} \\
(79.188 .8)\end{array}$ & $\begin{array}{l}(35.0 \\
(35-53.0)\end{array}$ & $\begin{array}{l}958.8 \\
(95.599 .7)\end{array}$ & $\begin{array}{c}8.8 \\
(80.3-90.2)\end{array}$ & $\begin{array}{l}6.1 \\
(4.3-8.8)\end{array}$ & $\begin{array}{c}62.5 \\
(139-280.6)\end{array}$ & $\begin{array}{l}0.94 \\
(0.882-0.996)\end{array}$ \\
\hline
\end{tabular}

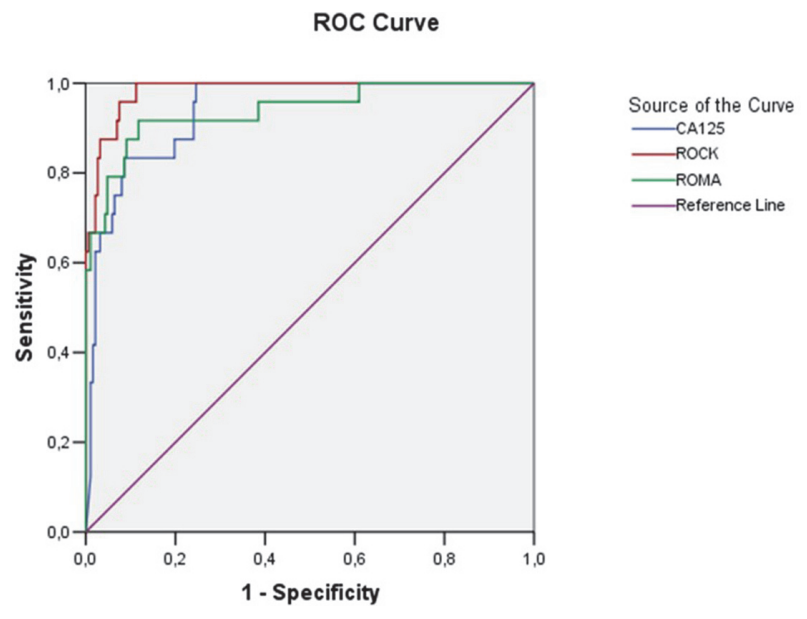

Abstract 1128 Figure 1 The ROC-curves of ROCK-I, ROMA and CA125 in the validating dataset
Conclusion* The proposed ROCK-I has demonstrated greater diagnostic performance than both ROMA and CA125 in the analyzed dataset. If an independent validation will show similar or even slightly lower difference between ROCK-I and ROMA it may provide a new basis of routine-use of HE4 in premenopausal patients with pelvic mass.

\section{UTERINE METASTASIS OF BREAST CANCER}

${ }^{1} \mathrm{D}$ Dhib*, ${ }^{2} Y$ Fertani, ${ }^{1} \mathrm{~F}$ Mghirbi, ${ }^{2} \mathrm{~L}$ Naija, ${ }^{1} \mathrm{~A}$ Gabsi, ${ }^{1} \mathrm{~A}$ Mokrani, ${ }^{1} \mathrm{~F}$ Ksontini, ${ }^{1} \mathrm{M}$ Ayadi, ${ }^{1} Y$ Yahyaoui, ${ }^{2} \mathrm{~K}$ Ben Ralah, ${ }^{1}$ A Mezlini. Institut Salah Azaiez, Medical Oncology, Tunis, Tunisia; ${ }^{2}$ Institut Salah Azaiez, Surgical Oncology, Tunis, Tunisia

\subsection{6/ijgc-2021-ESG0.647}

Introduction/Background* Uterine metastasis from extravaginal cancers is a rare event. A few cases of lobular invasive carcinoma with endometrial metastasis was reported in the litterature.

Result(s)* A 40 year old woman was treated in 2015 for a localised synchronous bilateral invasive ductal breast carcinoma. Human epidermal growth factor receptor (HER2) was amplified with positive endocrine receptor. She underwent in 2015 a neoadjuvant sequential hemotherapywith trastuzumab, followed by bilateral mastectomy, adjuvant trastuzumab and hormonotherapy (Tamoxifene with chemical ovarian suppression). In 2020, the CT scan showedliver, bone and peritoneal metastasis associated with a retro uterine mass suggesting a cervical cancer as a second cancer.

A biopsie was performed and the morphological feature with immunohistochemistry concluded to a metastasis of ductal breast carcinoma : Gata 3 protein(+), $\mathrm{ER}+, \mathrm{PR}+$, HER2 overexpressed and Wt1 negative .

Conclusion* Although rare, intrauterine breast cancer metastasis can occur even with a ductal breast cancer and physicians must go for a biopsy with immunohistochemistry.

\section{Endometrial cancer}

\section{MULTIPLEX QPCR HOTSPOT TESTING OF PATHOGENIC POLE MUTATIONS: A RAPID, SIMPLE AND RELIABLE APPROACH FOR POLE ASSESSMENT IN ENDOMETRIAL CANCER}

${ }^{1}$ AS Van den Heerik*, ${ }^{2} \mathrm{~N}$ Ter Haar, ${ }^{1} \mathrm{~N}$ Horeweg, ${ }^{2} \mathrm{~T}$ Van Wezel, ${ }^{1} \mathrm{C}$ Creutzberg, ${ }^{2} \mathrm{~V}$ Smit, ${ }^{2} \mathrm{~T}$ Bosse. 'Leiden University Medical Center (LUMC), Radiation Oncology, Leiden, Netherlands; 'Leiden University Medical Center (LUMC), Pathology, Leiden, Netherlands

\subsection{6/ijgc-2021-ESGO.648}

Introduction/Background* Detection of pathogenic somatic variants in the exonuclease domain (EDM) of the POLE-gene is of prognostic importance because of the excellent clinical outcomes of POLE-mutated endometrial cancers (EC). It is hypothesised that patients with POLE-mutated EC benefit of treatment de-escalation. Since $8-10 \%$ of EC carry a pathogenic POLE-mutation, it is of strong clinical importance to accurately determine the presence of these mutations. In current practice $P O L E$-status can only be determined by DNAsequencing methods, e.g. Sanger or Next-Generation-Sequencing (NGS). These techniques require a molecular biologist for 
correct interpretation, are relatively time consuming, not widely available and/or expensive. Due to the long turnaround time, it can be challenging to fit POLE-testing in tight timelines of clinical practice. To overcome this we have developed, and are in the process of validating, a rapid, simple, reliable and low-cost quantitative polymerase-chain-reaction (qPCR) assay for pathogenic POLE-mutations.

Methodology Primer and fluorescence-labelled 5'-nuclease probe-sequences of the five most frequently occurring pathogenic variations within exons 9,13 and 14 of the POLE EDM have been developed and optimized using DNA extracted from formalin-fixed paraffin-embedded tumour tissues. The simplicity of the design enables POLE-status assessment within 4 hours. Ongoing calibration studies are evaluating the minimal amount and quality of DNA required. Result(s)* Cut offs for failed, POLE-negative and -positive results were predefined based on 50 POLE-wildtypes and 6 $P O L E$-mutated cases. In the range of uncertainty in between, NGS-testing is recommended. In our first testing set of 115 cases (40 POLE-mutated, 75 POLE-wildtype), two samples failed, (one POLE-mutant, one wildtype) possibly due to low DNA quality. Four samples fell in the range of uncertainty (three POLE-mutant, one wildtype). Almost all POLE-mutants (33/36) were positive with no false-positives. Sensitivity and specificity were 92\% (95\%CI 83\%-100\%) and 100\%. Posthoc adjusment of the lower cut-off yielded a sensitivity of 97\% (95\%CI 92\%-100\%) and specifity of 100\%.

Conclusion* With this qPCR assay we developed a faster and simple alternative for targeted NGS-sequencing of the five most common pathogenic POLE-mutations. The assay-s simplicity in design and methods will make universal low-cost POLE-testing available for all EC patients.

\section{THE IMPACT OF INTENSIVE FOLLOW-UP ON HEALTH- RELATED QUALITY OF LIFE AND COSTS IN ENDOMETRIAL CANCER PATIENTS: DATA FROM THE TOTEM TRIAL NCT00916708}

${ }^{1} \mathrm{P}$ Zola*, ${ }^{2} \mathrm{G}$ Ciccone, ${ }^{3} \mathrm{E}$ Piovano, ${ }^{4} \mathrm{~L}$ Fuso, ${ }^{1} \mathrm{E}$ Peirano, ${ }^{5} \mathrm{DDI}$ Cuonzo, ${ }^{5} \mathrm{E}$ Pagano, ${ }^{5} \mathrm{~A}$ Castiglione, ${ }^{6} \mathrm{AM}$ Perrone, ${ }^{7} \mathrm{VD}$ Mandato, ${ }^{8} \mathrm{~L}$ Zavallone, ${ }^{9} \mathrm{~F}$ Chiudinelli, ${ }^{10} \mathrm{R}$ Berretta, ${ }^{11} \mathrm{~S}$ Loda, ${ }^{12} \mathrm{M}$ Valenzano Menada, ${ }^{13} \mathrm{~S}$ Greggi, ${ }^{14} \mathrm{M}$ Adorni, ${ }^{15} \mathrm{G}$ Comerci, ${ }^{16} \mathrm{M}$ Fambrini, ${ }^{4}$ A Ferrero. ${ }^{1}$ University of Turin, Department of Surgical Sciences, Turin, Italy; ${ }^{2}$ Città della Salute e della Scienza, University of Turin, Cancer Epidemiology Unit, CeRMS and C.P.O. Piemonte, Turin, Italy; ${ }^{3}$ A.O.U. Città della Salute e della Scienza di Torino, S. Anna Hospital, Obstetrics and Gynecology Unit n 3, Turin, Italy; ${ }^{4}$ A.S.O. Ordine Mauriziano, Obstetrics and Gynecology Unit, Turin, Italy; ${ }^{5}$ A.O.U. Città della Salute e della Scienza di Torino and CPO Piemonte, Clinical Epidemiology, Turin, Italy; ' S.Orsola-Malpighi Hospital, Gynecologic Oncology Unit, Bologna, Italy; ${ }^{7}$ A.O. Arcispedale S. Maria Nuova, Obstetrics and Gynecology Unit, Reggio Emilia, Italy; ${ }^{8}$ Infermi Hospital, Department Medical Oncology, Biella, Italy, ${ }^{9}$ P. O. Manerbio, A.S.S.T. Garda, Obstetrics and Gynecology Unit, Manerbio, Italy; ${ }^{10}$ Azienda Ospedaliero Universitaria di Parma, U.O.C. Ginecologia e Ostetricia, Parma, Italy; ${ }^{11}$ A.O. Spedali Civili, Obstetrics and Gynecology Unit, Brescia, Italy; ${ }^{12}$ Azienda Ospedaliera Universitaria San Martino, Obstetrics and Gynecology Unit, Genoa, Italy; ${ }^{13}$ Istituto Tumori Fondazione G. Pascale, MITO and Gynecologic Oncology, Naples, Italy; ${ }^{14}$ A.S.O. San Gerardo de' Tintori, Obstetrics and Gynecology Unit, Monza, Italy; ${ }^{15}$ Ospedale "Santa Maria delle Croci", U.O.C. di Ostetricia e Ginecologia, Ravenna, Italy; ${ }^{16}$ University of Florence, Department of Biomedical, Experimental and Clinical Sciences, Division of Obstetrics and Gynecology, Florence, Italy

\subsection{6/ijgc-2021-ESG0.649}

Introduction/Background* Intensive follow-up (FU) in cancer patients can be a source of stress for patients and absorbs a lot of health system resources. The TOTEM study was planned to compare an intensive (INT) vs minimalist (MIN) 5-year FU regimen in endometrial cancer patients in terms of overall survival (OS), health-related-quality-of-life (HRQL) and costs. This report focuses on HRQL and costs.

Methodology Patients surgically treated for endometrial cancer, FIGO stage I-IV, were stratified by center and in low (LoR) or high (HiR) risk of recurrence and then randomized to INT or MIN hospital-based FU regimens. HRQL was assessed at baseline-6-12 months and then yearly (with the SF-12 Physical and Mental Health Summary Scale). For per-participant average estimates, costs were tallied for each participant and divided by FU regimen to evaluate the difference between the two regimens. Bootstrapping was used for Confidence Intervals (CI) of cost differences between the FU regimens.

Result(s)* 1871 patients were randomized in 42 centers, and 1847 patients were available for the final analysis (60\% LoR). No difference in terms of OS was observed. Compliance with the FU scheduled visits was $75.3 \%$, similar between INT (74.7\%) and MIN (75.9\%) arms. In LoR the MIN arm performed slightly more procedures than expected (5080 vs $4955)$ and the INT arm performed fewer tests than expected (7505 vs 8830 ). In the HiR the MIN arm performed approximately the number of procedures expected (3621 vs 4004), while the INT arm performed far fewer exams than expected (7655 vs 11681). HRQL did not differ between arms (figures 1 and 2). In LoR patients, the mean real cost of INT arm was 698 euro vs 273 euro for the MIN arm. In HiR patients, the mean real cost of INT arm was 1116 euro vs 632 euro for the MIN arm. The estimated cost difference between the two FU regimens was 447.20 euro (95\% CI 398.87-494.97 euro).

Conclusion* In endometrial cancer treated patients the regimen of FU has no impact on HRQL. For both LoR and HiR patients there is a cost-saving with the MIN approach of about 450 euro per patient.
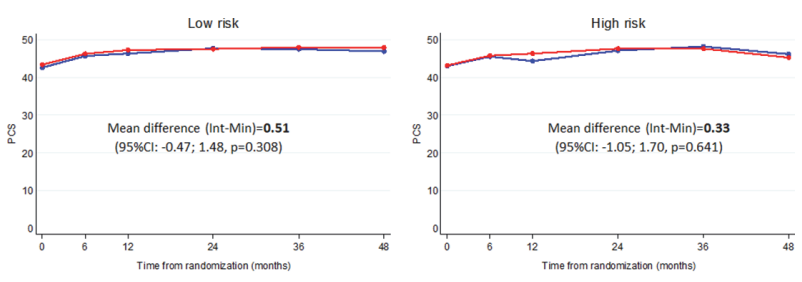

Abstract 259 Figure 1 HRQL_SF12-physical component summary, by risk

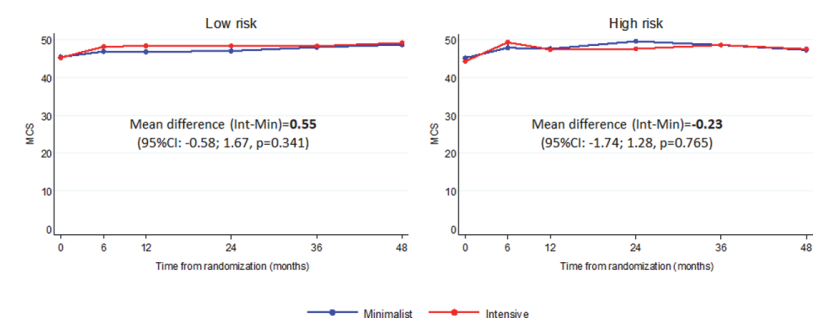

Abstract 259 Figure 2 HRQL_SF12-mental component summary, by risk 